Neurosurg Focus 19 (4):Intro, 2005

\title{
Bioinformatics in medical practice
}

\author{
STEPhen J. Haines, M.D. \\ Department of Neurosurgery, University of Minnesota Medical School, Minneapolis, Minnesota
}

As scientific disciplines grow and develop new methods of investigation and enter new areas of inquiry, the changes that follow sometimes lead to the definition of a new field. The process is not an orderly one; there is no moment of birth for a new scientific discipline. The unifying concepts are changing as rapidly as they are defined. The boundaries resemble those of eighteenth-century Europe more than twentieth-century America, with frequent changes, annexations, rebellions, and secessions. It is in this state that we find the field of "bioinformatics."

Derived from the French term bioinformatique (computer science for biological data), it encompasses, at times, complex analytic techniques for large amounts of data in genomics and proteomics and large database analysis of clinical data collected from insurance companies. Bioinformatic investigations may be as abstract as the development of theoretical models and neural networks or as concrete as defining the structure of DNA. They may look at populations as large as that of the world or as small as the DNA of a single organism.

To quote from a definition promulgated by the National Institutes of Health:

The NIH Biomedical Information Science and Technology Initiative Consortium agreed on the following definitions of bioinformatics and computational biology recognizing that no definition could completely eliminate overlap with other activities or preclude variations in interpretation by different individuals and organizations.

Bioinformatics: Research, development, or application of computational tools and approaches for expanding the use of biological, medical, behavioral or health data, including those to acquire, store, organize, archive, analyze, or visualize such data.

Computational Biology: The development and application of data-analytical and theoretical methods, mathematical modeling and computational simulation techniques.
(http://www.bisti.nih.gov/CompuBioDef.pdf [Accessed 11 October 2005]).

Many if not all areas of bioinformatics investigation and application apply to neurosurgery. The genomics and proteomics of central nervous system disease are areas of intense investigation, and advances in these fields may well lead to interventional therapies for diseases that until now have been the province of the watchful waiting of medical neurology. Neural network development and computational systems biology offer understanding of the complex workings of higher intellectual function that may have profound implications for neurosurgical intervention. The massive data manipulations required for functional magnetic resonance (MR) imaging, diffusion tensor imaging, spectroscopy, and future MR diagnostic techniques have already profoundly changed the practice of neurosurgery. The more mundane but no less important clarity of understanding of the value of clinical interventions with large-scale database investigations can guide clinical neurosurgical decision making to a much more scientific and effective mode of practice than has been possible up to this time.

It is also important to recognize that the methods of bioinformatics are under constant revision and development. There is art as well as science in medicine, and the sophisticated consumer of medical publications needs to be well informed enough about methods to analyze the offered information critically and skeptically incorporate it into daily practice.

In this issue of Neurosurgical Focus we can only suggest the possibilities that bioinformatics offers to the neurosurgery of the future. It is a daunting and exciting future, and progress requires that neurosurgeons be familiar with and use these techniques. 